\title{
ANALYSIS OF THE TIME EVOLUTION OF TEMPORAL COHERENCE USING COSMO-SKYMED HH AND VV DATA
}

\author{
Balbarani, Sebastián ${ }^{(1,2)}$; Euillades, Pablo A. ${ }^{(1,2)}$; Euillades, Leonardo D. ${ }^{(1,2)}$; Riveros, Natalia C. $^{(1)}$ \\ (1) CEDIAC Institute - National University of Cuyo, Argentina, sbalbarani@cediac.uncu.edu.ar \\ (2) National Scientific and Technical Research Council-CONICET, Argentina
}

\begin{abstract}
Physical changes that occur in land surfaces between two SAR acquisitions affect the precision of repeatpass synthetic aperture radar differential interferometry (DInSAR). This phenomena is defined by the temporal term of interferometric coherence value in each interferometric pair. In this work we carried out an empirical study of the COSMO SkyMed (X-band) temporal decorrelation in both polarization modes, $\mathrm{HH}$ and VV. We analysed the polarization effect on the temporal coherence in relation with: 1) percentage of coherent pixels in the regions of interest (ROI), and 2) the seasonal behaviour over the vegetated areas.
\end{abstract}

\section{INTRODUCTION}

Differential SAR Interferometry (DInSAR) is a geodetic technique that allows investigating terrain-deformationrelated geophysical processes. It takes profit from the phase difference between two SAR scenes in order to estimate surface motion achieving sub-centimetre to sub-millimetre precision [1]. A great deal of phenomena can be studied by using this technique, including active tectonics, earthquakes, urban subsidence, ground water, oil and gas exploitation, volcanism, landslides and anthropogenic structures like tunnels and mining facilities [2].

European Space Agency (ESA) SAR Missions ERS-1/2 and ENVISAT provided over two decades of useful data for DInSAR. Availability of a great number of scenes repeatedly acquired over Areas of Interests (AOIs) promoted the development of the so called time-series techniques (MT-InSAR) which allow investigating the deformation history of every coherent pixel. Most popular between them are Small Baseline Subsets (SBAS) [3] and Persistent Scatterers [4]. SBAS makes profit of distributed scatterers by using multilooked interferograms in order to enhance the SNR ratio. Spatial and temporal decorrelation is minimized by selecting interferometric pairs characterized by small spatial and temporal baseline. By the contrary, PS solves for point scatterers. It generates full resolution interferograms between the available scenes and a common master one. As a consequence, high density of coherent points is achieved in favourable areas, like urbanized ones.

Key parameter that gives an idea of the phase quality in an interferogram is the interferometric coherence. It is approximated from the data by means of a maximum likelihood estimator [5], and takes values between 0 and 1. Thus, the phase corrected coherence is estimated as [6]:

$$
|\tilde{\gamma}|=\frac{\left|\sum_{n=1}^{N} z_{1}^{(n)} z_{2}{ }^{*(n)} e^{-j \phi^{(n)}}\right|}{\sqrt{\sum_{n=1}^{N}\left|z_{1}{ }^{(n)}\right|^{2} \sum_{n=1}^{N}\left|z_{2}{ }^{(n)}\right|^{2}}}
$$

Where $z_{1}$ and $z_{2}$ are both the SAR complex signals, $\phi^{(n)}$ is the systematic phase component for each pixel, and $N$ is the nominal number of looks. Temporal evolution of this parameter at a generic resolution cell is an indicator of the time this cell will allow computing reliable results. Furthermore, coherence is a measurement of phase error used for improving phase unwrapping [7].

Total coherence value depends not only on the scatterers properties but also on the acquisition geometry and other SAR system characteristics. A priori multiplicative model was proposed in [8]:

$$
\gamma_{\text {tot }}=\gamma_{\text {geom }} \cdot \gamma_{C D} \cdot \gamma_{\text {therm }} \cdot \gamma_{\text {temp }}
$$

Where $\gamma_{\text {geom }}$ is the geometric component related with perpendicular spatial baseline, $\gamma_{D C}$ is the Centroid Doppler difference contribution, $\gamma_{\text {therm }}$ is the thermal noise and $\gamma_{\text {temp }}$ is the temporal coherence.

On the other hand, a second generation SAR systems has been launched during the last years. X-band missions COSMO-SkyMed (ASI) and the TerraSAR-X (DLR), orbiting the Earth since 2007, are characterized by reduced revisit time and high spatial resolution. Increased availability of SAR data makes interesting investigating decorrelation with $\mathrm{X}$-band data and timeseries processing. In particular, COSMO-SkyMed constellation allow acquiring data with $\mathrm{HH}, \mathrm{VV}, \mathrm{HV}$ and $\mathrm{VH}$ polarizations [9]. The questions are: Which acquisition mode is more convenient for long term 
interferometric exploitation? Is decorrelation different at different polarization? Are coherent pixels in $\mathrm{HH}$ scenes the same as in VV ones?

Trying to answer those questions, we analysed the behaviour of the temporal coherence in a COSMO SkyMed dataset acquired by interleaving $\mathrm{HH}$ and $\mathrm{VV}$ acquisitions covering a common AOI. Temporal coherence depends on the changes in the individual scatterers inside the resolution cell. Those changes are due to scatterers re-distribution and/or re-orientation and change in dielectric properties of the observed surface. Scatterers re-distribution and re-orientation in a rural environment can be due to fields plowing and irrigation. Natural phenomena like vegetation growing, wind, rainfall, snowfall and temperature change introduce modification both in the relative location of scatterers and in the soil moisture content, leading to temporal decorrelation. The diversity of causes makes temporal coherence a magnitude not easily modelled. Reference [8] proposed a theoretical exponential model taking into account horizontal and vertical scatterers random movement. However, that model is not easily extrapolable to a real environment because individual scatterers displacement is not available in a general case.

In this work we present the results of an empirical study regarding temporal decorrelation occurred in $\mathrm{HH}$ and VV COSMO SkyMed time series.

\section{DATASETS AND STUDY AREA}

In this research we used two COSMO-SkyMed datasets which transmitter/receiver configuration was $\mathrm{HH}$ and VV. They were composed of $32 \mathrm{HH}$-polarized and 30 VV-polarized SAR images. The acquisition mode was the same for all images: Interferometric Stripmap (HIMAGE) in descending pass with an incidence angle of $38^{\circ}$. Both datasets cover the period between June 2010 and March 2012 (one and a half year), but HHand VV-polarized scenes were acquired interleaved in time, as can be seen in Fig. 1.

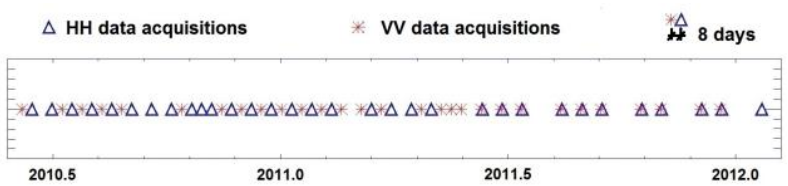

\section{Figure 1. Distribution of $H H$ and VV data acquisitions}

The Area of Interest is Mendoza city in western Argentina. It covers the Mendoza metropolitan area and surroundings including different coverage types: I) urban areas (Mendoza city), II) agricultural coverage around the city, III) piedmont and bare soil with low vegetation, and IV) high relief (Andes Mountains). Area location and main characteristics are presented in Fig. 2. The orange box identifies an approximation of the orbital descending footprint of $\mathrm{HH}$ and VV COSMOSkyMed dataset $(40 \times 50$ kilometres). The background image correspond to Landsat- 8 sensor of 23 May 2013 (path: 232, row: 83) available in U.S. Geological Survey website.

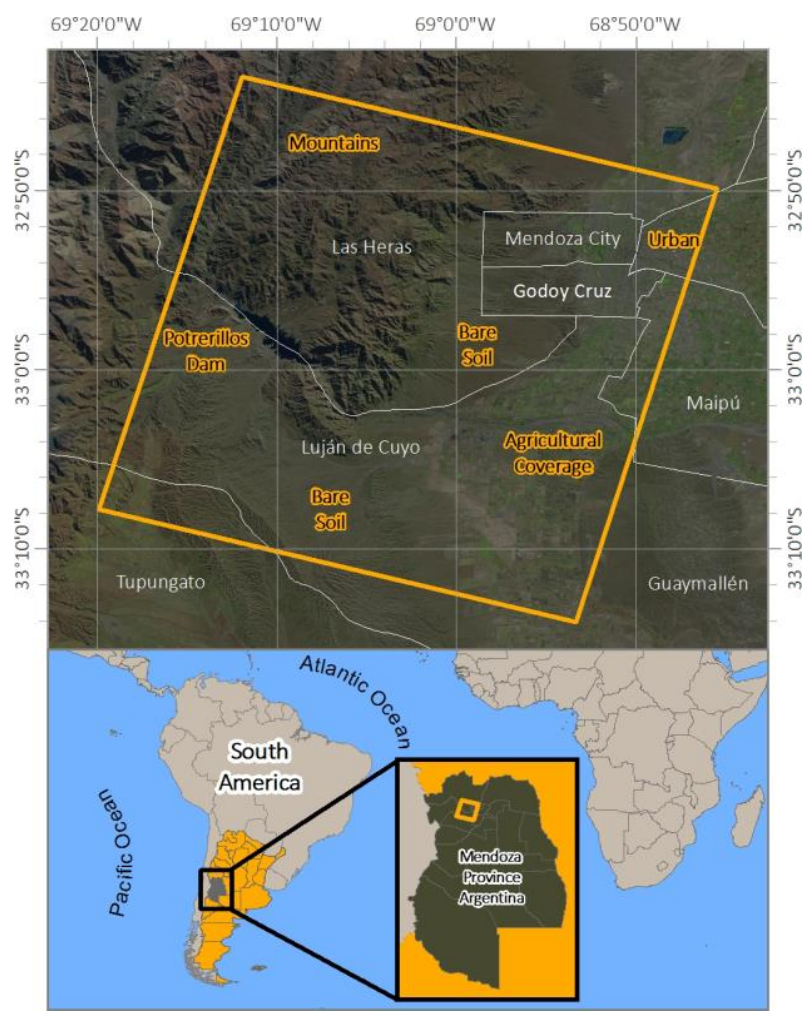

Figure 2. Study area: the city of Mendoza, Argentina.

\section{METHODOLOGY}

We start from COSMO SkyMed raw data (level 0): 32 HH-polarized and $30 \mathrm{VV}$-pol. We focused all SAR images and extracted the system parameters.

Accurate subpixel registration of scenes with respect to the reference master acquisition was carried out. Besides, we generated all possible interferometric products: $496 \mathrm{HH}-\mathrm{HH}$ and $435 \mathrm{VV}-\mathrm{VV}$ differentials interferograms and coherence maps. The $30 \mathrm{~m} \mathrm{X}$-band shuttle radar topography mission - SRTM digital elevation model [10] was used for the topographic phase removal.

On the other hand, we converted digital elevation model (DEM) to SAR coordinates: range and azimuth. We computed de incidence angle matrix from orbital and DEM coordinates, and we estimated the distance targetsensor in the processing master image geometry. 
In order to separate temporal decorrelation from geometrical one, we applied a simple but effective method proposed by [11]. Local slope in range direction was computed from incidence angle matrix, DEM and range matrix. Subsequently, we carried out the range and azimuth spectral shift estimation [12] in order to generate the geometrical coherence maps.

After estimating geometric coherence, temporal coherence can be obtained by simply dividing the observed coherence map by geometrical one from Eq 2 . In Fig. 3 we shown the block flow of de processing chain.

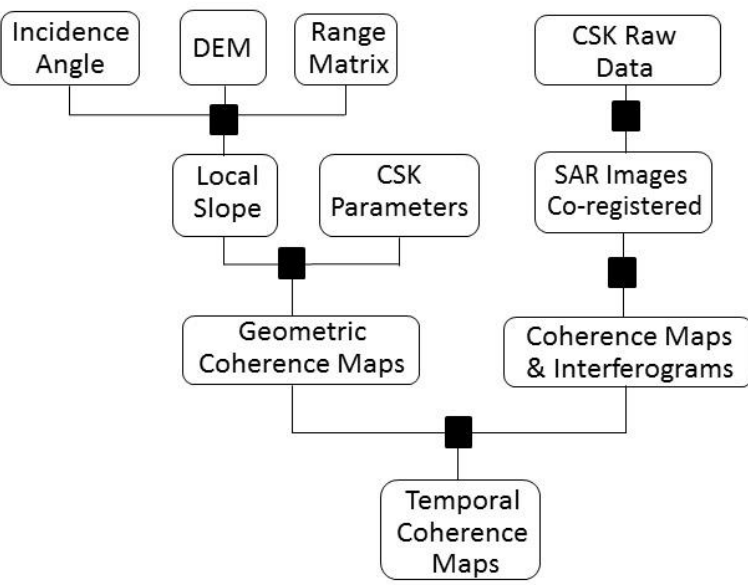

Figure 3. Block flow of Processing Chain

Thereby we generated $496 \mathrm{HH}-\mathrm{HH}$ polarized and 435 VV-VV-pol temporal coherence maps. COSMO SkyMed temporal decorrelation is the major contribution to total decorrelation. It is one of the main limitations of SAR interferometry and it depends of the temporal baseline, coverage types and weather situations.

In this work, we used temporal coherence values for analysing the percentage of coherent pixels inside the regions of interest, and its seasonal behaviour as a function of polarization in presence of vegetation,

\section{RESULTS}

Temporal coherence maps were studied by defining regions of interest (ROIs) relevant for different land coverage: 1) urban, 2) bare soil, 3) agricultural and 4) mountainous.

A pixel is considered coherent when its temporal coherence is higher than a defined threshold in a significant number of interferograms. For this study, we set the temporal coherence threshold at 0.25 and the percentage of interferograms to $30 \%$. This criteria is similar to the one employed in the SBAS implementations for deciding which pixels remain in the final solution [7].

\subsection{Coherent Pixels Rate}

Temporal coherence was analysed in the ROIs relevant for the soil coverage, as can be seen in Fig. 4. Urban area is marked with violet colour, bare soil with cyan, agricultural coverage with yellow and mountainous with red.

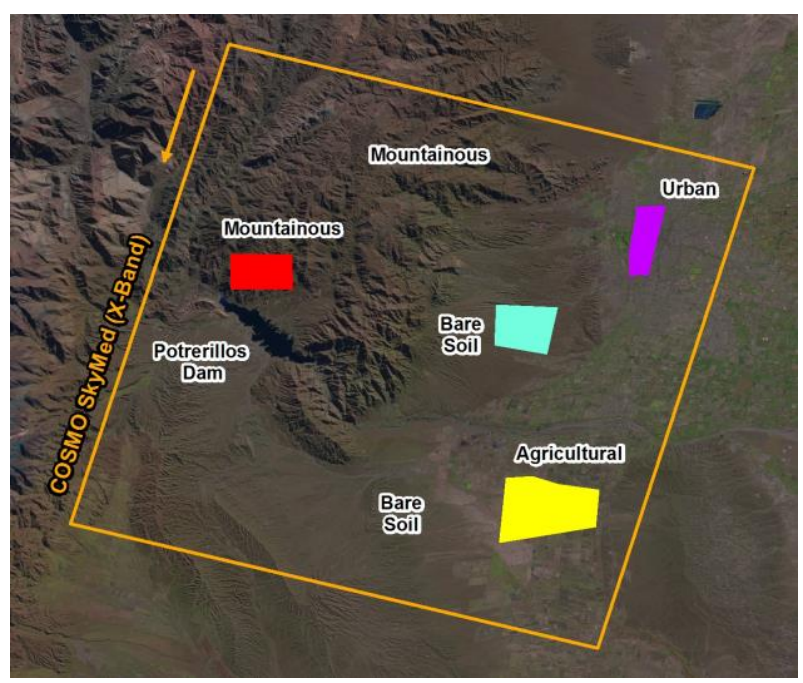

Figure 4. Region of interest defined

We considered all the pixels inside the ROI and we converted each one from geographic coordinates to SAR geometry (range/azimuth).

Table 1. Pixel status in term of temporal coherence

\begin{tabular}{|c|c|c|c|c|}
\hline $\begin{array}{c}\text { Soil } \\
\text { Coverage }\end{array}$ & Polarization & $\begin{array}{c}\text { Number of } \\
\text { Pixels (ROI) }\end{array}$ & Total & [\%] \\
\hline \multirow{4}{*}{ Urban } & Incoherent & 6837 & \multirow{4}{*}{31077} & 22.00 \\
\hline & Only in $\mathrm{HH}$ & 2175 & & 7.00 \\
\hline & Only in VV & 622 & & 2.00 \\
\hline & $\mathrm{HH}$ and VV & 21443 & & 69.00 \\
\hline \multirow{4}{*}{ Bare Soil } & Incoherent & 13034 & \multirow{4}{*}{43445} & 30.00 \\
\hline & Only in $\mathrm{HH}$ & 3910 & & 9.00 \\
\hline & Only in VV & 3041 & & 7.00 \\
\hline & $\mathrm{HH}$ and $\mathrm{VV}$ & 23460 & & 54.00 \\
\hline \multirow{4}{*}{ Mountainous } & Incoherent & 30329 & \multirow{4}{*}{40439} & 75.00 \\
\hline & Only in $\mathrm{HH}$ & 2022 & & 5.00 \\
\hline & Only in VV & 809 & & 2.00 \\
\hline & $\mathrm{HH}$ and VV & 7279 & & 18.00 \\
\hline
\end{tabular}


Table 1 shows the pixel status, in terms of temporal coherence, for data at different polarization inside the ROIs. As can be appreciated, vast majority of pixels show similar behaviour in $\mathrm{HH}$ and VV data: $91 \%$ in urban environment, $84 \%$ in bare soil, $93 \%$ in mountainous terrain and $\sim 100 \%$ in agricultural fields.

As expected, most pixels in agricultural ROI are incoherent in both polarizations. Mountainous terrain behaves differently because, despite it is composed of stable structures like rock outcrops, it receives heavy snowfall in winter, and so only $18 \%$ of pixels remain coherent according to the used criteria.

HH polarization shows a marginally higher ratio of coherent pixels when compared with VV polarization. This effect is observed in urban area, bare soil and mountainous environment.

\subsection{Seasonal Behaviour}

In order to analyse the behaviour in time of the temporal coherence, we categorized the coherence maps according to the time span between master and slave scenes. We generate temporal decorrelation maps subsets of identical temporal baseline: 16 days, 32 days, 48 days, and more.

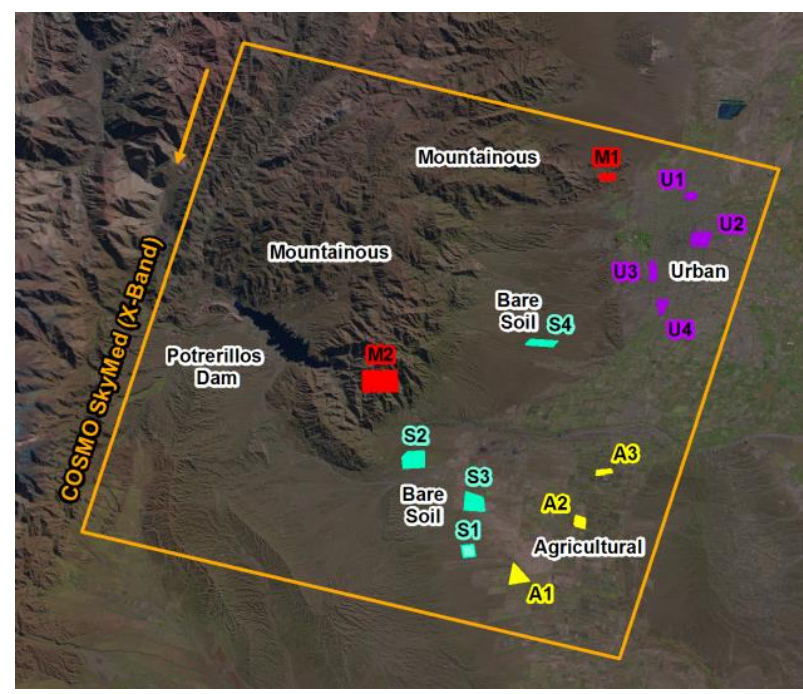

Figure 5. Regions of Interest for statistical analysis

Then, for each ROI presented in the Fig. 5 and time span, we computed the temporal coherence mean, median, mode, standard deviation, variance, skewness, kurtosis and minimum and maximum value.

Bare soil and agricultural coverage presents Gaussian distribution (symmetric and unimodal) with standard deviation 0.10 and 1.12 , respectively. On the other hand, urban area show high data dispersion and a positive skew.
One example is showed in the Fig. 6. It corresponds to CSK-HH temporal coherence distribution (Temporal baseline $=8$ days. Master image: 10/21/2010 - slave image: $10 / 29 / 2010$.

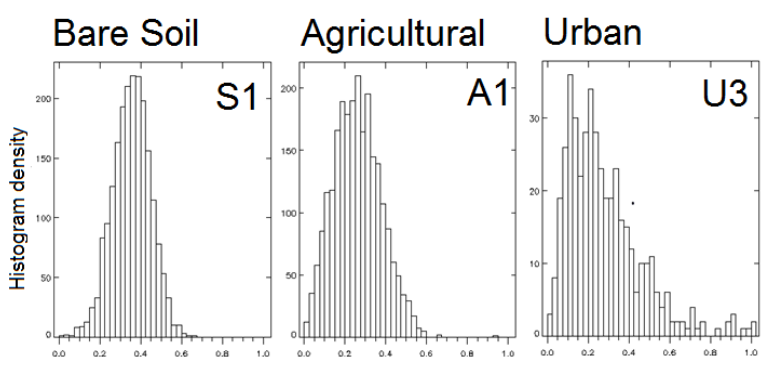

Figure 6. Histogram Density

Behaviour in time of temporal coherence as a function of the master date over agricultural area, bare soil and urban environment is presented in Fig. 7.

We represent temporal coherence value by means of box and whiskers plots. Figure shows the results for bare soil, urban and agricultural ROIs considering 16days time span as a function of master scene date.
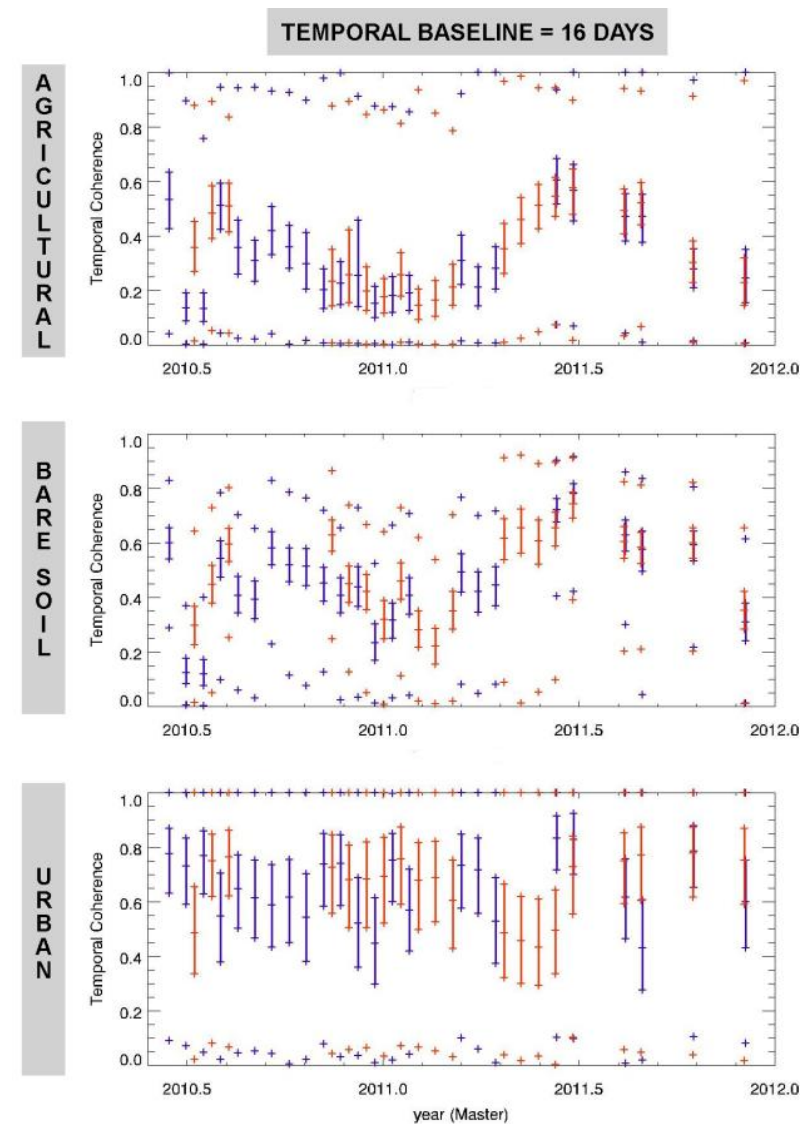

Figure 7. Temporal coherence behaviour in time. Blue: HH-pol. Red: VV-pol 
Seasonal behaviour in agricultural soil is noticeable when time span is 16 days. Temporal coherence reach peaks of $\sim 0.60$ in winter and drop to less than 0.20 in summer. This effect is not surprising because after the crop season (January to march) fields remain mostly undisturbed until the spring (September). Coherence rapidly increase from March to August and then drop towards the summer when the growing season peaks.

Two coherence maps in July 2010 show anomalous behaviour with temporal coherence of $\sim 0.10$. We suspect that they can represent short term coherence loss like the one provoked by snowfall. It remains as future work testing that hypothesis.

Bare soil areas behave similarly to the agricultural ones. Coherence is relatively high reaching peaks of $\sim 0.80$ during the winter whereas summer valleys are about 0.30. This effects can be due to growing of sparse natural vegetation located within these areas.
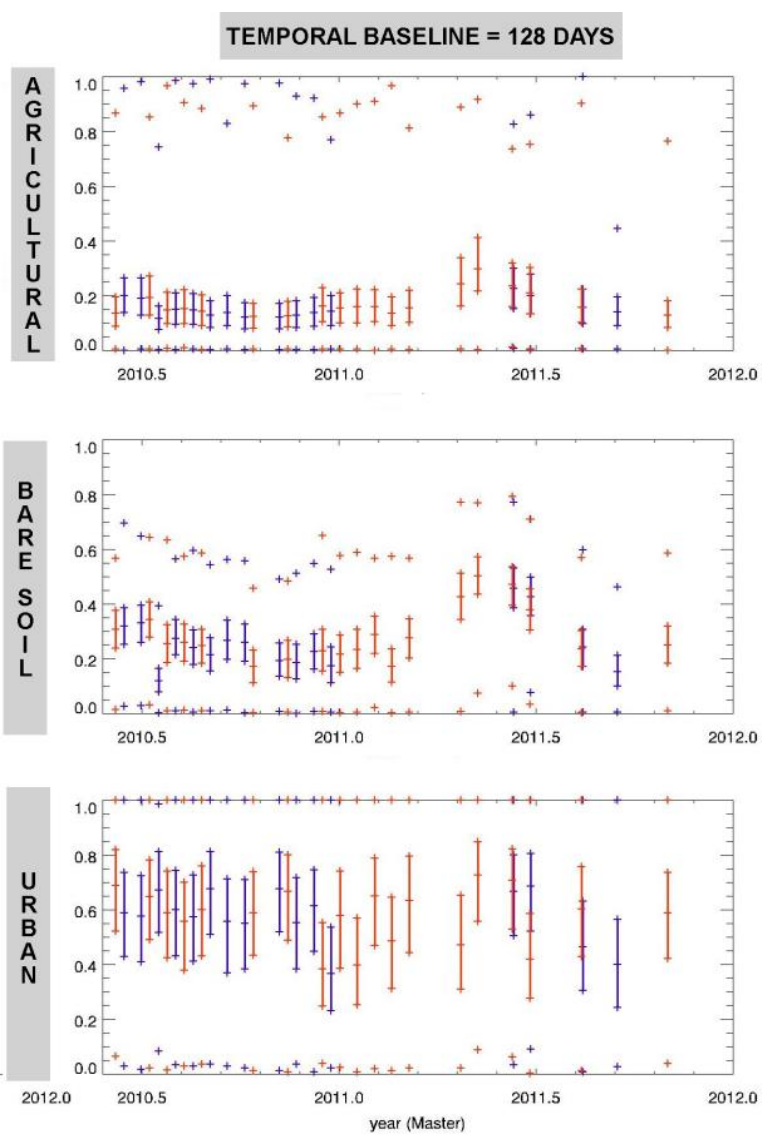

Figure 8. Temporal coherence behaviour in time. Blue: HH-pol. Red: VV-pol

The urban case is somewhat different. Temporal coherence at 16 days' time span is high and stable independently of the master scene date. However, we observe also higher dispersion of values than in the other ROIs. This effect could be due to the fact that in
Mendoza city the urban vegetation (trees) is really significant. Thus, temporal coherence in urban ROIs are a mixture of man-made structures coherence and deciduous trees coherence.

Behaviour in time of temporal coherence as a function of the master date when temporal baseline is 128 days is illustrated in Fig. 8.

When time span is set to 128 days (agricultural ROI) the seasonal behaviour is less noticeable. Winter peaks are still observed (0.25), but temporal coherence is around 0.15 most of the time. Same behaviour is observed in bare soil ROI, although coherence is always higher than in the agricultural case.

When 128 days' time span in urban area, a slight drop of coherence is observed, but it remains stable all year long.

\section{CONCLUSIONS}

We carried out an empirical study of temporal coherence. It is very dependent of land cover and for this reason we analysed it defining regions of interest. We investigated the influence of polarization in the temporal coherence behaviour in the deformation time series context.

In reference to the quantity of coherent pixels in the ROIs we observed similar $\mathrm{HH}$ and $\mathrm{VV}$ behaviour. Urban area and bare soils shows many coherent pixels with respect to agricultural and mountainous soil. Slightly higher number of coherent pixels in $\mathrm{HH}$ polarization can be observed also.

Regarding the X-band temporal decorrelation, a seasonal behaviour in presence of vegetation can be noted. One important observation relevant for all the analysed ROIs is that the temporal coherence show no significant difference when $\mathrm{HH}$ and $\mathrm{VV}$ polarized scenes are studied.

\section{REFERENCES}

1. Gabriel, A.K., Goldstein, R.M., Zebker, H.A. (1989). Mapping small elevation changes over large areas: differential radar interferometry. J. Geophys. Res. Solid Earth. 1978-2012 94, 9183-9191.

2. Massonnet, D. y K. Feigl (1998). Radar Interferometry and its Application to Changes in the Earth's Surface. Reviews of Geophysics. Vol 36, No 4, pp. 441-500.

3. Berardino, P., Fornaro, G., Lanari, R., Sansosti, E. (2002). A new algorithm for surface deformation monitoring based on small baseline differential SAR interferograms. IEEE Trans. Geosci. Remote 
Sens. 40, 2375-2383.

4. Ferretti, A., Prati, C., Rocca, F. (2001). Permanent scatterers in SAR interferometry. Geosci. Rem. Sens. IEEE. On 39, 8-20.

5. Seymour, M. S. and Cumming, I. G. (1994). Maximum Likelyhood Estimation For SAR Interferometry. International Geoscience and Remote Sensing Symposium, Pasadena, CA, USA, 8-12 August 1994, pp. 2272-2275.

6. Hagberg, J. O., Ulander, L. M. H. and Askne, J. (1995). Repeat-Pass SAR Interferometry over Forested Terrain, IEEE Transactions on Geoscience and Remote Sensing, 33(2):331-340.

7. Pepe, A., Lanari, R. (2006). On the extension of the minimum cost flow algorithm for phase unwrapping of multitemporal differential SAR interferograms. Geosci. Remote Sens. IEEE Trans. On 44, 2374-2383.

8. Zebker, H.A., Villasenor, J. (1992). Decorrelation in interferometric radar echoes. Geosci. Remote Sens. IEEE Trans. On 30, 950-959.

9. COSMO SkyMed System (2010). Description and Userguide: http://www.e-geos.it/products/pdf/cskuser_guide.pdf. Last access: 04/02/2015.

10. Rabus, B., Eineder, M., Roth, A., and Bamler, R. (2003). The Shuttle Radar Topography Mission - a New Class of Digital Elevation Models Acquired by Spaceborne Radar, ISPRS J. Photogramm. Remote Sens., 57, 241-262, 2003.

11. Wang, T., Liao, M., Perissin, D. (2010). InSAR Coherence-Decomposition Analysis. IEEE Geosci. Rem. Sens. Let. 7, 156-160.

12. Gatelli, F., Monti Guarnieri, A., Parizzi, F., Pasquali, P., Prati, C. and Rocca, F. (1994). The wavenumber shift in SAR Interferometry, IEEE Transactions on Geoscience and Remote Sensing, 32(4):855-865.

13. Gatelli, F., Monti Guarnieri, A., Parizzi, F., Pasquali, P., Prati, C. and Rocca, F. (1994). The wavenumber shift in SAR Interferometry, IEEE Transactions on Geoscience and Remote Sensing, 32(4):855-865. 